\title{
ARISTOLOCHIA OCCIDENTALIS (ARISTOLOCHIACEAE), UNA ESPECIE NUEVA \\ DE LA SUBSECCION PENTANDRAE DE COLIMA Y JALISCO, MEXICO
}

\author{
Francisco J. Santana-Michel \\ Instituto Manantlán de Ecología \\ y Conservación de la Biodiversidad \\ Centro Universitario Costa Sur \\ Universidad de Guadalajara \\ Independencia Nacional No. 151 \\ Apartado Postal 64 \\ 48900 Autlán, Jalisco, México \\ Y \\ Sebastian Lemus Juarez \\ Centro Universitario de Investigación \\ y Desarrollo Agropecuario \\ Universidad de Colima \\ Crucero Estación Tecomán \\ Carretera Colima-Manzanillo km 43 \\ 28100 Tecomán, Colima, México
}

\section{RESUMEN}

Se describe Aristolochia occidentalis sp. nov. procedente de la región limítrofe entre los estados de Colima y Jalisco. La especie pertenece a la subsección Pentandrae Duchartre y está relacionada con Aristolochia pringlei Rose y Aristolochia bracteosa Duchartre.

\section{ABSTRACT}

Aristolochia occidentalis sp. nov. is described from near the border between the Mexican states of Colima and Jalisco. The species is a member of the subsection Pentandrae Duchartre. It is related to Aristolochia pringlei Rose and Aristolochia bracteosa Duchartre.

El género Aristolochia L. fue dividido por Duchartre (1854) en 5 secciones, de acuerdo con el número de estambres y lóbulos de la columna del ginostemo. La sección Gymnolobus Duchartre, la divide el mismo autor en dos subsecciones: Pentandrae Duchartre y Hexandrae Duchartre, con 5 y 6 estambres y lóbulos estigmáticos respectivamente. La subsección Pentandrae prácticamente se distribuye en casi todo México, con 35 especies conocidas (Pfeifer, 1970); para los estados de Jalisco y Colima 
se han registrado 12 (Santana-Michel, 1993), además de la que aquí se propone como nueva, cuya descripción es la siguiente:

Aristolochia occidentalis Santana-Michel et S. Lemus sp. nov. Fig. 1.

Herbae perennes scandentes raro reptantes, pilosae 1-3 m longae; folia petiolata, sagittata, subcordata, apice acuminata, supra glabra vel sparsim pilosa, pagina subtiliter papillosa, subtus tomentosa; petioli (1.3)2-3(-4) $\mathrm{cm}$ longi, 0.5-0.7 mm diametro; lamina (2.5-)8-13 cm longa, (1-)3.5-4.5(-8.5) cm lata; flores axillares solitarii, pedunculis bracteolatis, bracteola lanceolato cordata, (1.5-)2-3(-3.7) cm longa, (0.5-)1-1.5(-2) cm lata; calyx rectilinearis lanceolatus, viridis pallidus fauce purpurea, hypanthio inconspicuo, utriculo ovoideo 7-8 mm longo, 4-5 mm diametro, syringe excentrica, 1.8-2 mm longa sensim angustata, tubo recto, piloso, (6-)7-9(-10) $\mathrm{mm}$ longo, 3-4 mm diametro, limbo lanceolato, 1-lobato, basi cordato, apicem versus acuto, piloso, subtiliter tuberculoso, (3-)3.5-4.7(-5) cm longo; columna 5-lobata, substipitata, 3-4 mm longa, 2.5-2.6 mm diametro, staminibus 5, tetraloculatis; capsula botuliformis, 2-2.5 lata, 1.5-2 cm diametro, 5-carpellata, basi dehiscens, septifraga; semina numerosa, triangulata, $4-5 \mathrm{~mm}$ longa, 3.8-4.9 $\mathrm{mm}$ lata, 0.5-0.7 mm crassa, nigra, superficie subtiliter tuberculata.

Hierbas perennes, trepadoras, rara vez rastreras, de 1-3 m de largo; hojas pecioladas, sagitado-subcordadas, ápice acuminado, haz glabro o con pelos dispersos, la superficie finamente papilosa, el envés tomentoso, pecíolo de (1.3-)2-3(-4) cm de largo por 0.5-0.7 mm de diámetro, lámina de (2.5-)8-13 cm de largo, (1-)3.5-4.5(-8.5) cm de ancho; flores axilares, solitarias, el pedúnculo bracteolado, de $(0.6-) 0.8-1.5(-1.7) \mathrm{cm}$ de largo, 0.6-1 mm de diámetro, bracteolas lanceolado-cordadas, de (1.5-)2-3(-3.7) cm de largo, (0.5-)1-1.5(-2) cm de ancho, cáliz rectilineo, lanceolado de color verde claro y la garganta púrpura, hipantio no evidente, utrículo ovoide, de 7-8 $\mathrm{mm}$ de largo, 4-5 mm de diámetro, el siringe excéntrico, de 1.8-2 mm de largo, angostándose al terminar, tubo recto, piloso, de (6-)7-9(-10) mm de largo, 3-4 mm de diámetro, limbo lanceolado, 1-lobado, agudo hacia el ápice, cordado en la base, piloso, finamente tuberculado, de (3-)3.5-4.7(-5) cm de largo, (0.5-)0.6-1.3(-1.5) cm de ancho; ginostemo 5-lobado, subestipitado, de 3-4 mm de largo, 2.5-2.6 $\mathrm{mm}$ de diámetro, estambres 5, tetraloculares. Fruto una cápsula botuliforme, 5-carpelar, de dehiscencia basicida, septifraga, de $2-2.5 \mathrm{~cm}$ de largo, $1.5-2$ $\mathrm{cm}$ de diámetro, semillas negras, numerosas, triangulares, de 4-5 mm de largo, 3.8-4.9 $\mathrm{mm}$ de ancho, 0.6-0.8 $\mathrm{mm}$ de grueso, la superficie finamente tuberculada.

HOLOTIPO: México, Colima, entre El Poblado y Peña Colorada, municipio de Minatitlán (límite entre Colima y Jalisco), $1000 \mathrm{~m}$ de altitud, en bosque tropical caducifolio, 18 de octubre de 1992, F. J. Santana-M. y S. Lemus J. 6206 (ZEA); Isotipos en ENCB, IBUG, MEXU, WIS.

Material adicional examinado: México, Colima, $1 \mathrm{~km}$ al E de Peña Colorada, $4 \mathrm{~km}$ al NW de Minatitlán, municipio Minatitlán, $1200 \mathrm{~m}$ de altitud, bosque tropical caducifolio, 29 octubre 1992, F. J. Santana-M. y S. Lemus J. 6045 (ZEA); Jalisco, 6 km al SW de La Huerta, municipio de La Huerta, $600 \mathrm{~m}$ de altitud, bosque tropical subcaducifolio, 26 de abril de 1992, F. J. Santana-M. 5645 (en fruto ZEA, IBUG). 
Santana-Michel et al.: Aristolochia occidentalis, Una Especie Nueva de Colima y Jalisco

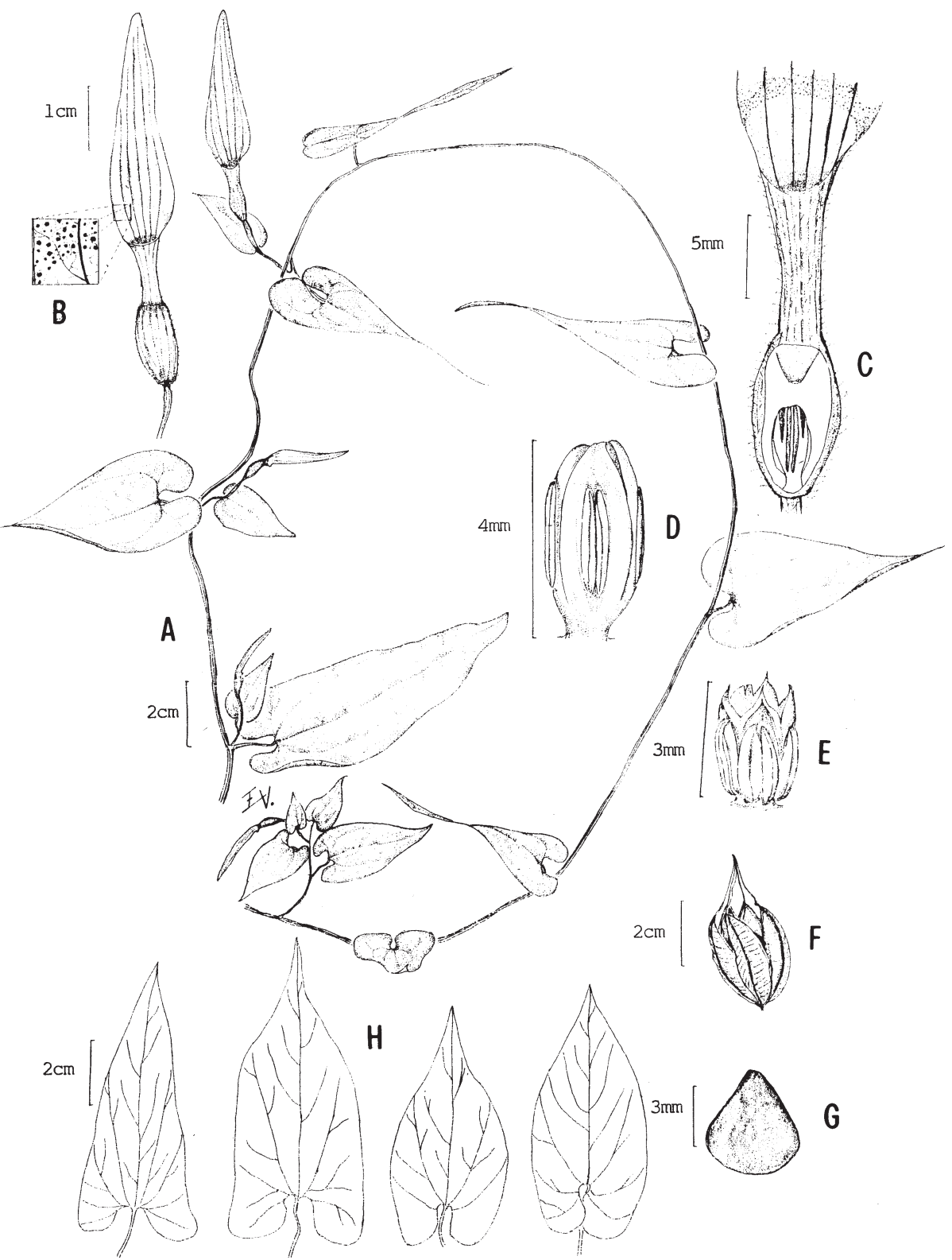

Fig. 1. Aristolochia occidentalis. A. planta; B. detalle de una parte del limbo; C. detalle mostrando el siringe y ginostemo; D. ginostemo maduro; E. ginostemo inmaturo; F. fruto; G. semilla; H. variación de la hoja. llustración basada en la colección tipo (Santana-M. y S. Lemus J. 6206), elaborada por Enrique V. Sánchez Rodríguez. 
Distribución conocida: México, estados de Colima y Jalisco.

Epoca de floración: de septiembre a noviembre.

Habitat: Bosque tropical caducifolio en suelos delgados, pedregosos con pediente pronunciada y la presencia de las siguientes especies arbóreas: Lysiloma acapulcense, L. microphyllum, Pseudobombax ellipticum, Bursera arborea, B. copallifera, B. fagaroides, B. bipinnata, Pseudosmodingium perniciosum, Plumeria rubra, Thouinia serrata, Trichilia americana, Leucaena macrophylla, Ceiba aesculifolia. Bosque tropical subcaducifolio predominando las especies arbóreas: Brosimum alicastrum, Annona purpurea, A. reticulata, Acacia glomerosa, Tabebuia rosea, Hura polyandra, Trichostigma mexicanum, Lysiloma microphyllum.

Aristolochia occidentalis pertenece a la subsección Pentandrae; presenta flores rectilineas, el tubo más corto que el limbo, hojas anchas, pecioladas y parece estar relacionada con Aristolochia pringlei Rose y Aristolochia bracteosa Duchr., las cuales pueden separarse fácilmente (ver Cuadro 1).

Cuadro 1. Caracteres de Aristolochia occidentalis y especies afines.

\begin{tabular}{|c|c|c|c|}
\hline & A. pringlei & A. bracteosa & A. occidentalis \\
\hline Posición y crecimiento & voluble & procumbente & voluble \\
\hline Lámina foliar & sagitado-hastada & $\begin{array}{l}\text { ovada a triangular- } \\
\text { lanceolada }\end{array}$ & $\begin{array}{l}\text { sagitado-subcor- } \\
\text { dada }\end{array}$ \\
\hline Pecíolo & $1.5-4.5 \mathrm{~cm}$ & $3-5 \mathrm{~mm}$ & $1.3-4 \mathrm{~cm}$ \\
\hline Flor & verde-amarillenta & purpúrea & verde clara \\
\hline Limbo & $\begin{array}{l}\text { lanceolado-acumi- } \\
\text { nado } \\
4-6.5 \mathrm{~cm}\end{array}$ & $\begin{array}{l}\text { ovado-lanceolado } \\
2-4 \mathrm{~cm}\end{array}$ & $\begin{array}{l}\text { lanceolado-agudo } \\
3-4.7 \mathrm{~cm}\end{array}$ \\
\hline
\end{tabular}

\section{AGRADECIMIENTOS}

Gracias a las monografías elaboradas por el Dr. Howard W. Pfeifer sobre el género Aristolochia, ha sido posible realizar este trabajo. Al Instituto Manantlán de Ecología y Conservación de la Biodiversidad y al Departamento de Botánica de la Universidad de Wisconsin se reconoce el apoyo obtenido para el estudio de la flora de la región, especialmente al Dr. Hugh H. Iltis, Enrique J. Jardel Peláez, Bruce F. Benz y Luz Ma. Villarreal de Puga. Al Ing. Roberto González Tamayo se dan las gracias por la revisión 
y sugerencias al manuscrito. $Y$ finalmente el primer autor agradece a sus compañeros colaboradores en el inventario y estudio de la flora de la Sierra de Manantlán: J. Antonio Vázquez G., Ramón Cuevas G., Luis Guzmán H., Nora M. Nuñez L., Leticia Hernández L., E. Valente Sánchez R., Theodore Cochrane, Mark Wetter, Judith Cevallos E., Elizabeth Muñoz M. y Jesús J. Rosales A.

\section{LITERATURA CITADA}

Duchartre, P. 1854. Tentamen methodicae divisionis generis Aristolochia. Annales des Sciences Naturelles. Paris 54: 29-33.

Pfeifer, H.W. 1970. A revision of the pentandrous species of Aristolochia. The University of Connecticut Publication Series. Storrs, Connecticut. 134 pp.

Santana-Michel, F. J. 1993. Revisión taxonómica del género Aristolochia L. (Aristolochiaceae) en los estados de Jalisco y Colima, México. XII Congreso Mexicano de Botánica. Resumen. Mérida, Yucatán. p. 243. 